\title{
Collisional particle simulation of ion acoustic instability
}

\author{
R. D. SY DOR A ${ }^{1}$, F. DETER I G G ${ }^{2}$, W. R OZM U S ${ }^{1}$, \\ Y. Y U. B Y CHENKOV ${ }^{3}$, A. B R A N T O ${ }^{1}$ and C. E. CA P J A C K \\ ${ }^{1}$ Department of Physies, University of Alberta, Edmonton, AB, Canada T6G 2J1 \\ ${ }^{2}$ Centre de Physique Theorique, Ecole Polytechnique, Palaiseau 91128, France \\ ${ }^{3}$ P.N. Lebedev Physics Institute, Russian Academy of Sciences, Leninskii prospect 53, \\ Moscow 119991, Russia \\ ${ }^{4}$ Department of Electrical and Computer Engineering, University of Alberta, Edmonton, \\ $\mathrm{AB}$, Canada T6G 2J1
}

(Received 31 August 2005 and accepted 8 June 2006)

\begin{abstract}
The nonlinear evolution of ion acoustic fluctuations in the presence of a uniform electron drift which exceeds the sound speed and electron-ion collisions is investigated using a collisional particle simulation model for parameters relevant to laser-heated plasmas. The results indicate that for the one-dimensional case, strong electron-ion collisionality can lead to a significantly higher saturation level of the fluctuations, which is comparable to the saturation level in the two-dimensional collisionless regime. This relatively high turbulence level can act to enhance heat flux inhibition and strongly influence the absorption and transport properties of laser-produced plasmas.
\end{abstract}

\section{Introduction}

Electron heat transport is a central topic in the field of laser-plasma interaction physics and in inertial confinement fusion. In many cases the laser energy absorption occurs on very short scales and involves temperature gradients which are too steep for the classical Spitzer-Härm Coulomb transport theory to be applicable (non-local regime). It has been demonstrated theoretically and through kinetic simulations (Fokker-Planck and particle) that non-locality leads to inhibited electron transport [1, 2]. Another possible mechanism for the electron heat conductivity is associated with ion acoustic turbulence [3]. It has been shown that the fast electrons that conduct thermal energy away from a hot plasma region also carry an electric charge which must be compensated for by the return current of cold electrons from the bulk of the distribution. This return current drives ion acoustic waves if the drift velocity, $V_{\mathrm{De}} \approx q_{\mathrm{e}} / n_{\mathrm{e}} T_{\mathrm{e}}$, exceeds the sound speed, $c_{\mathrm{s}}=\left(Z T_{\mathrm{e}} / m_{\mathrm{i}}\right)^{1 / 2}$, where $q_{\mathrm{e}}$ is the electron heat flux and $n_{\mathrm{e}}$ and $T_{\mathrm{e}}$ are the local electron number density and temperature, respectively. It has been recently shown that the heat flux changes the shape of the electron distribution such that it becomes a more effective driver of ion acoustic turbulence and for most laser-plasma interaction experiments the threshold conditions for instability are significantly exceeded [4].

We have recently investigated non-local electron heat transport in the presence of return current-driven ion acoustic fluctuations for the physical scenario of localized hot spots arising from inhomogeneous laser intensity distributions of randomized 
laser beams [5]. The observed growth of the return current instability was in excellent agreement with the predictions of a linear, nonlocal theory [6].

Our previous work was limited to a one-dimensional (1D) hot spot geometry and therefore only ion acoustic fluctuations with a single wave vector parallel to the thermal gradient were included. Oblique ion acoustic modes have a large growth rate and therefore we have extended our model to two-dimensional. The purpose of this paper is to illustrate the influences of electron-ion collisions and twodimensional (2D) effects on the saturation dynamics of ion acoustic fluctuations within the homogeneous electron drift model, as a first step.

\section{Kinetic ion acoustic mode}

The linear properties of the ion acoustic instability for a weakly inhomogeneous plasma with net electron drift, $V_{\text {De }}$, are summarized. For an electron and ion plasma with Maxwellian distributions the real frequency is given by

$$
\omega_{k}=\omega_{\text {pi }} k \lambda_{\text {De }} /\left(1+k^{2} \lambda_{\text {De }}^{2}\right)^{1 / 2}
$$

and growth rate

$$
\begin{aligned}
\gamma_{k} \approx & \sqrt{\frac{\pi}{8}} \frac{\omega_{\mathrm{pi}}}{\left(1+k^{2} \lambda_{\mathrm{De}}^{2}\right)^{2}}\left[\left(\frac{Z m_{\mathrm{e}}}{m_{\mathrm{i}}}\right)^{1 / 2}\left(\frac{\hat{k} \cdot \mathbf{V}_{\mathrm{De}}}{c_{\mathrm{s}}}\left(1+k^{2} \lambda_{\mathrm{De}}^{2}\right)^{1 / 2}-1\right)\right. \\
& \left.-\left(\frac{Z T_{\mathrm{e}}}{T_{\mathrm{i}}}\right)^{3 / 2} \exp \left(-\frac{1}{2} \frac{Z T_{\mathrm{e}} / T_{\mathrm{i}}}{1+k^{2} \lambda_{\mathrm{De}}^{2}}-\frac{3}{2}\right)\right],
\end{aligned}
$$

where $\omega_{\text {pi }}=\omega_{\text {pe }}\left(Z m_{\mathrm{e}} / m_{\mathrm{i}}\right)^{1 / 2}$ and $\hat{k}=\mathbf{k} /|\mathbf{k}|$. The ion modes are unstable $\left(\gamma_{k}>0\right)$ for $\hat{k} \cdot \mathbf{V}_{\text {De }}>c_{\mathrm{s}} /\left(1+k^{2} \lambda_{\text {De }}^{2}\right)^{1 / 2}$ when $Z T_{\mathrm{e}} / T_{\mathrm{i}} \gg 1$. The most unstable wavelength is peaked around $k \lambda_{\mathrm{De}} \approx 0.7$ and the growth rate maximizes for oblique modes with the angle of propagation $\theta \approx \cos ^{-1}\left(c_{\mathrm{s}} / V_{\mathrm{De}}\right)$. In local theory, with temperature inhomogeneity, the drift speed is proportional to the temperature gradient and inversely proportional to the electron-ion collision frequency [7].

\section{Simulation model and results}

We use a kinetic simulation model based on the particle-in-cell (PIC) approach to describe inhomogeneous, collisional laser plasma heating and collective plasma response. The ions and electrons are evolved using particle dynamics consistent with the 2D collisionless Vlasov equation. Electron-ion collisions are introduced using a Monte Carlo representation of the Fokker-Planck collisional operator in the Lorentz approximation [5].

The simulation parameters are chosen to be consistent with laser-produced plasma experiments $\left(T_{\mathrm{e}}=530 \mathrm{eV}, T_{\mathrm{i}}=53 \mathrm{eV}, n_{\mathrm{e}}=10^{20} \mathrm{~cm}^{-3}\right)$. The normalized parameters used in the simulations were $T_{\mathrm{e}} / T_{\mathrm{i}}=10, Z=10, \nu_{\mathrm{ei}} / \omega_{\mathrm{pe}}=0.05, V_{\mathrm{De}} /$ $c_{\mathrm{s}}=2.3$ and $m_{\mathrm{i}} / m_{\mathrm{e}}=200$. The mass ratio was chosen to be small to speed up the computation and scalings have been done up to the value of 20000 . The numerical parameters consisted of system size $L_{x}=64 \lambda_{\text {De }}$ for the $1 \mathrm{D}$ case and $L_{x} \times L_{y}=64 \lambda_{\mathrm{De}} \times 64 \lambda_{\mathrm{De}}$ for the $2 \mathrm{D}$ case with cell size $\Delta x=\Delta y=\lambda_{\text {De }}$. The total number of particles per cell corresponded to 50000 for the $1 \mathrm{D}$ case and 5000 for the $2 \mathrm{D}$ case and time step $\omega_{\mathrm{pe}} \Delta t=0.1$ was used.

The results of both $1 \mathrm{D}$ and $2 \mathrm{D}$ cases are presented. In Fig. 1 the total electrostatic energy as a function of time is displayed for both the collisionless (1D, 2D) and collisional (1D) regimes. For the 1D cases, electron-ion collisions reduce the linear 


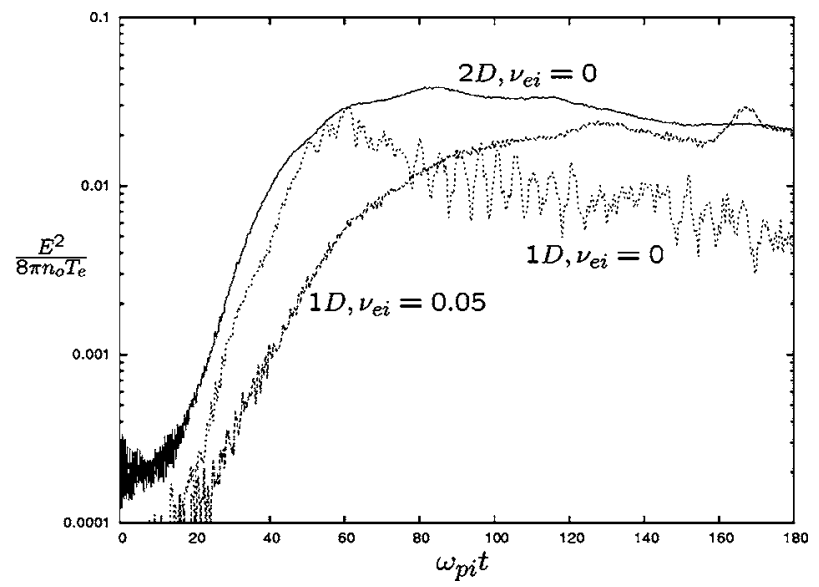

Figure 1. Evolution of the total electrostatic energy for the 1D case with and without collisions and the collisionless $2 \mathrm{D}$ system.

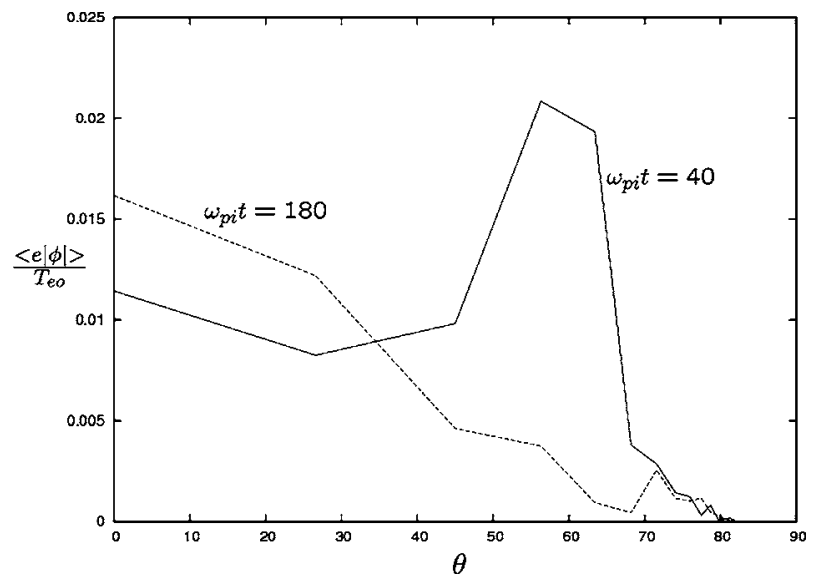

Figure 2. Electrostatic fluctuation amplitude versus mode angle with respect to the electron drift at time levels corresponding to the linear phase (solid curve) and the saturation phase (dashed curve). Averaging in time is made over 10 time steps.

growth rate, however, in the saturation phase the fluctuation energy remains higher by about a factor of five (with larger differences when smaller mass ratios are used). This is mainly due to the isotropization of the electron distribution in the collisional case which maintains the instability drive by preventing the quasilinear relaxation of the electron distribution at the wave phase speed [8]. The $2 \mathrm{D}$ case with collisions (not shown) did not significantly differ in saturation amplitude compared with the collisionless case displayed here. This is possibly due to the nature of the saturation processes in the $1 \mathrm{D}$ and $2 \mathrm{D}$ situations.

In the $2 \mathrm{D}$ case the unstable ion acoustic wave spectrum consists of oblique modes which lead to a higher level of fluctuations as compared with the 1D collisionless case. The linear phase is dominated by modes with angle of propagation corresponding to $\theta \approx \cos ^{-1}\left(c_{\mathrm{s}} / V_{\mathrm{De}}\right) \approx 64^{\circ}$ as illustrated in Fig. 2 . In the saturation phase, the fluctuations spread in cone angle and peak at angles less than $40^{\circ}$. Figure 3 shows the time evolution of the oblique mode corresponding to $\theta=59^{\circ}$ which reduces in amplitude at later times as the wave energy scatters to longer wavelengths. 


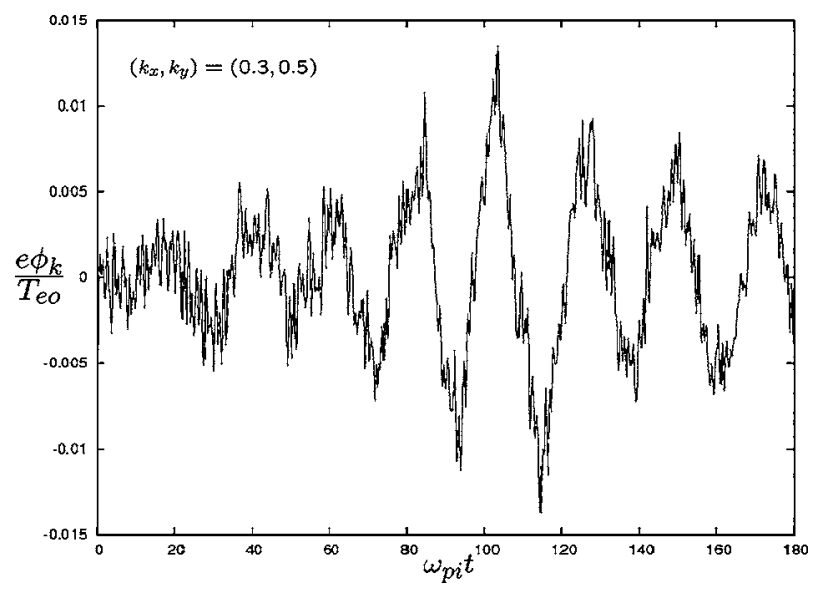

Figure 3. Evolution of the electrostatic potential (real part) for the mode angle corresponding to $\theta=59^{\circ}$ or $\left(k_{x}, k_{y}\right)=(0.3,0.5)$.

\section{Summary}

The nonlinear growth and saturation dynamics of the kinetic ion acoustic instability, excited by a uniform electron drift, have been investigated in one and two dimensions for parameters relevant to laser-produced plasmas. It has been shown that electron-ion collisions can lead to enhanced fluctuation levels in the saturation phase of the instability even though the linear drive is weakened. Furthermore, the inclusion of oblique ion acoustic modes in $2 \mathrm{D}$ simulations leads to a much broader spectrum and higher levels of turbulence as compared with the 1D collisionless regime. The consequences of the $2 \mathrm{D}$ effects on absorption and transport are currently being investigated for the hot spot configuration considered in [5].

\section{References}

[1] Batischev, O., Bychenkov, V. Yu., Detering, F., Rozmus, W., Sydora, R. D., Capjack, C. E. and Novikov, V. N. 2002 Heat transport and electron distribution function in laser produced plasmas with hot spots. Phys. Plasmas 9, 2302-2310.

[2] Brunner, S. and Valeo, E. 2002 Simulations of electron transport in laser hot spots. Phys. Plasmas 9, 923-936.

[3] Estabrook, K. 1981 Hot electrons due to laser absorption by ion acoustic turbulence. Phys. Rev. Lett. 47, 1396-1399.

[4] Tikhonchuk, V. T., Rozmus, W., Bychenkov, V. Yu., Capjack, C. E. and Epperlein, E. 1995 Return current instability in laser heated plasmas. Phys. Plasmas 2, 4169-4173.

[5] Detering, F., Rozmus, W., Brantov, A., Bychenkov, V. Yu., Capjack, C. E. and Sydora, R. D. 2005 Particle-in-cell simulations of heat flux driven ion acoustic instability. Phys. Plasmas 12,012321.

[6] Brantov, A., Bychenkov, V. Yu. and Rozmus, W. 2001 Ion acoustic instability driven by a temperature gradient in laser-produced plasmas. Phys. Plasmas 8, 3558-3564.

[7] Monchicourt, P. 1983 Dynamics of the heat flux-induced ion turbulence in a laserproduced plasma. Phys. Fluids 26, 3354-3360.

[8] Dum, C. T., Chodura, R. and Biskamp, D. 1974 Turbulent heating and quenching of the ion sound instability. Phys. Rev. Lett. 32, 1231-1234. 